\title{
Flora apícola de la zona estepa espinosa Montano Bajo, en la Estación Experimental Tunshi, Riobamba, Ecuador
}

\section{Apicultural flora of the thorny steppe zone Montano Bajo, in the Tunshi Experimental Station, Riobamba, Ecuador}

\section{Apicultural flora da espinhosa zona de estepe Montano Bajo, na estação experimental de Tunshi, Riobamba, Equador}

Miguel Ángel Guallpa-Calva ${ }^{\mathrm{I}}$

miguel.guallpa@espoch.edu.ec

Edmundo Danilo Guilcapi-Pacheco II

eguilcapi@espoch.edu.ec

Armando Esteban Espinoza-Espinoza ${ }^{\text {III }}$

armando.espinoza@espoch.edu.ec

Recibido: 15 de diciembre de 2018 *Aceptado: 22 de febrero de 2019 * Publicado: 05 de abril de 2019

I Magíster en Formulación, Evaluación y Gerencia de Proyectos para el Desarrollo, Magíster en Manejo Forestal Sostenible, Ingeniero Forestal, Tecnólogo Agroforestal, Docente Escuela Politécnica de Chimborazo ESPOCH, Riobamba, Ecuador.

II Magíster en Producción Animal, Diploma Superior en Investigación y Proyectos, Ingeniero Agrónomo, Docente Escuela Politécnica de Chimborazo ESPOCH, Riobamba, Ecuador.

III Máster en Ciencias Mención Agricultura Sustentable, Ingeniero Agrónomo, Docente Escuela Politécnica de Chimborazo ESPOCH, Riobamba, Ecuador. 


\title{
Resumen
}

En este estudio se evalúa la situación actual sobre la disponibilidad de la flora melífera existente en la Estación Experimental Tunshi, para conocer las especies vegetales con potencial apícola, y su abundancia con la finalidad de programar actividades de manejo sostenibles. La investigación se desarrolló con base en la delimitación del área de muestreo, la revisión de información reportada en la literatura especializada, la identificación taxonómica de especies vegetales, mediante la observación directa de las plantas en el sito y su verificación en material especializado, complementado con el registro de su hábito de crecimiento, más la estimación del nivel de abundancia de la presencia de cada especie vegetal por unidad de uso de manejo del predio. En la investigación se generó un listado de 32 especies vegetales, pertenecientes a 17 familias botánicas. Se distingue la familia Fabaceae con 11 especies, Asteraceae con 3, seguida por las familias; Myrtaceae, Rosaceae y Solanaceae con dos especies. Así mismo la abundancia en el área de cultivos, resultó con más de 10.000 individuos con flores de Raphanus spp., y entre 1.000 a 10.000 individuos Lupinus pubescens y Zea mays, con similar valor se determinó a Bidens andicola y Eucalyptus globulus presentes en los sistemas de usos; rastrojo y bosque plantado respectivamente. Aún es imperante complementar el estudio con datos de abundancia y su época e intensidad de floración afín a las abejas, por un periodo de al menos un año con el propósito de poder plantear estrategias de manejo que influyen en la sostenibilidad de la producción apícola.

Palabras clave: Apicultura; flora melífera; estado de floración; colmenar.

\begin{abstract}
In this study, the current situation regarding the availability of the existing honey flora in the Estació is evaluated. Experimental Tunshi, to know the plant species with apicultural potential, and their abundance with the purpose of programming sustainable management activities. The research was developed based on the delimitation of the sampling area, the review of information reported in the specialized literature, the taxonomic identification of plant species, through the direct observation of the plants in the site and their verification in specialized material, supplemented with the registration
\end{abstract}


of their habit of growth, plus the estimate of the level of abundance of the presence of each plant species per unit of land use. In the research, a list of 32 plant species belonging to 17 botanical families was generated. The family Fabaceae is distinguished with 11 species, Asteraceae with 3, followed by families; Myrtaceae, Rosaceae and Solanaceae with two species. Likewise, the abundance in the area of crops, resulted with more than 10,000 individuals with flowers of Raphanus spp., And between 1,000 to 10,000 individuals Lupinus pubescens and Zea mays, with similar value was determined to Bidens andicola and Eucalyptus globulus present in the systems of uses; stubble and forest planted respectively. It is still imperative to complement the study with abundance data and its time and intensity of flowering related to bees, for a period of at least one year with the purpose of being able to propose management strategies that influence the sustainability of apiculture production.

Key words: Beekeeping, honey flora, flowering status, apiary

\section{Resumo}

Neste estudo, a situação atual da disponibilidade de flora melífera existentes na Estação Experimental Tunshi é avaliada a conhecer as espécies vegetais com potencial de apicultura, e sua abundância, a fim de programar as atividades de manejo sustentável. A pesquisa foi desenvolvido com base na delimitação da área de amostragem, a avaliação da informação relatada na literatura, identificar as espécies de plantas por observação directa de plantas no local e a sua verificação no material especializado, suplementado por o registro de seu hábito de crescimento, mais a estimativa do nível de abundância da presença de cada espécie de planta por unidade de uso da terra. Na pesquisa, uma lista de 32 espécies de plantas pertencentes a 17 famílias botânicas foi gerada. A família Fabaceae é distinguida com 11 espécies, Asteraceae com 3, seguidas pelas famílias; Myrtaceae, Rosaceae e Solanaceae com duas espécies. Da mesma forma abundância na área de cultivo, foi mais do que 10.000 indivíduos com flores Raphanus spp., E 1.000 a 10.000 indivíduos pubescens Lupinus e Zea mays, com valor semelhante foi determinada Bidens andicola e Eucalyptus globulus sistemas actuais de usos; restolho e floresta plantada respectivamente. Ele ainda é prevalente complementar o estudo com dados de abundância e de tempo e intensidade de floração semelhante para as abelhas, por um período 
de pelo menos um ano, a fim de ser capaz de levantar estratégias de gestão que influenciam a sustentabilidade da apicultura.

Palavras-chave: Apicultura; flora do mel; estado florido; apiário

\section{Introducción}

Ecuador es un país mega diverso, en el cual existe una variación de clima (verano e invierno), humedad relativa, presión atmosférica, entre otros factores que permiten el crecimiento de variadas especies de plantas melíferas en las distintas regiones del país como lo es Costa, Sierra y Oriente (Vivas , 2015). Los emprendimientos apícolas a nivel de nuestro país y la provincia de Chimborazo, se encuentran en general a un nivel medio con técnicas orientadas a un manejo sostenible con el objeto de obtener una buena producción (Instituto Nacional de Tecnología Agropecuaria, 2018).

La apicultura es una actividad que aprovecha la vegetación, tanto en su estado natural como alterado, así como los cultivos agrícolas, pastizales, entre otros. Siendo la flora melífera uno de los pilares básicos que sustenta la actividad apícola y cuyo conocimiento de cada región en particular, la época, duración de su floración y su valor relativo como fuentes de néctar, polen o ambas sustancias a la vez, es indispensable para lograr buenos resultados en la producción de miel en escala comercial (Sánchez, 1995). La flora apícola es la que define la alternativa productiva de subproductos y pone límites a la producción, dependiendo de ella las características del producto. $\mathrm{Su}$ conocimiento es fundamental para la conducción racional del apiario, ya que constituye el recurso con que cuentan las abejas para alimentarse y producir. Así mismo, brinda información para determinar pautas de manejo del apiario en general y aún del campo en que se encuentra ubicado el colmenar (Ocaña y Ocaña, 2008).

Al reconocer e identificar el tipo de vegetación predominante, época de floración y la interrelación con los microclimas existentes, se podrán establecer condiciones que permitan mejorar las características organolépticas de las distintas mieles estableciendo así índices de calidad tanto en color, sabor como el aroma, al mismo tiempo se puede establecer las relaciones integrales entre estos índices de calidad (Heywood y Watson, 1995). Para conocer posibles necesidades de conservación y de restauración de los ecosistemas a fin de adaptar el manejo de los apiarios a los cambios en el 
potencial natural, es importante tener un buen conocimiento sobre la oferta floral como insumo para optimizar la producción, diferenciar productos de la colmena y mejorar la competividad (Silva y Restrepo, 2012).

\section{Materiales y Métodos}

La presente investigación se realizó en los sistemas agroecológicos identificados en la Estación Experimental Tunshi de la Escuela Superior Politécnica de Chimborazo, situada en Tunshi Grande, parroquia Licto, al sur oeste del cantón Riobamba, provincia de Chimborazo-Ecuador, a $12 \mathrm{~km}$ de la ciudad capital. Geográficamente la Estación Experimental se inserta dentro de los puntos de referencia: UTM Zona 17S Datum WGS 84 X1 = 763609; Y1 = 9806880, X=9806791, X3 = 764973; Y3 $=9805248$ y X4 =763606; Y =9805251. Se encuentra localizada a una altitud que va desde 2755 - $2929 \mathrm{msnm}$. La precipitación anual es $635,4 \mathrm{~mm}$, siendo la temperatura media $14,4^{\circ} \mathrm{C}$ y una humedad relativa media de 85 \%. Datos de la Estación Guaslan; serie 1990-2016; MAGAP. La Estación Experimental tiene una superficie total de 145 has y se categoriza dentro de la formación: estepa Espinoza Montano Bajo (Sierra et al., 2012).

La investigación se realizó en las áreas circundantes distantes entre 600 a $800 \mathrm{~m}$ hasta un apiario, a partir del punto de referencia: UTM Zona 17S Datum WGS 84, según se expone en el Punto de referencia para monitoreo de vegetación melífera, en la Tabla 1, que a continuación se presenta:

Tabla 1. Punto de referencia para monitoreo de vegetación melífera

\begin{tabular}{|c|c|c|}
\hline X & Y & Altitud (msnm) \\
\hline 763887 & 9806432 & 2735 \\
\hline
\end{tabular}

Fuente: Apuntes de Registro de campo. Elaboración: propia.

\section{Métodos}

En la investigación se aplicaron los métodos: documental, observacional y de campo, definidas para la identificación de plantas melíferas (Calvache, 2016). La identificación de las plantas apícolas tuvo lugar durante el segundo semestre del año 2018. Mediante la ejecución de las siguientes actividades: 


\section{Delimitación de las áreas de muestreo}

Con tres visitas de campo al sitio en estudio, mediante observación directa, el registro de coordenadas y cálculo de áreas con GPS, se tipificaron las zonas al presentar seis usos de suelo identificados en el predio, con base en los criterios que permitieron obtener la distribución de la superficie evaluada que se indica en la tabla 1.

Tabla 1. Distribución de usos de suelo en la Estación Experimental Tunshi

\begin{tabular}{|c|c|c|c|}
\hline Categoría & Descripción & Has & $\%$ \\
\hline Bosques plantados & $\begin{array}{l}\text { Especies foráneas de rápido crecimiento, plantadas } \\
\text { para la producción, comercialización o conservación }\end{array}$ & 42,05 & 29 \\
\hline Arbustos & $\begin{array}{l}\text { Vegetación arbustiva con menos de } 5 \mathrm{~m} \text { de altura que } \\
\text { se encuentra formando congregados de especies } \\
\text { vegetales o no }\end{array}$ & 7,25 & 5 \\
\hline Rastrojo & $\begin{array}{l}\text { Vegetación herbácea o leñosa que nace por } \\
\text { regeneración natural. De poca altura, máximo } 2 \text { metros }\end{array}$ & 2,9 & 2 \\
\hline Pastos & Pasturas naturales o plantadas & 46,4 & 32 \\
\hline Cultivos & $\begin{array}{l}\text { Vegetación compuesta por especies de uso agrícola y } \\
\text { forestal. Los cultivos pueden ser permanentes o } \\
\text { temporales }\end{array}$ & 36,25 & 25 \\
\hline Sistemas agroforestales & $\begin{array}{l}\text { Conjunto de diferentes especies de plantas con fines } \\
\text { específicos que constituyen arreglos. Un ejemplo son } \\
\text { las huertas o sistemas agroforestales. }\end{array}$ & 6,15 & 4 \\
\hline \multirow[t]{2}{*}{ Bordes de caminos } & $\begin{array}{l}\text { Aquellos márgenes adyacentes a las vías que posee el } \\
\text { predio }\end{array}$ & 4 & 3 \\
\hline & Total & 145 & 100 \\
\hline
\end{tabular}

Fuente: Guión de observación directa aplicada por los investigadores. Elaboración: propia

\section{Monitoreo e identificación taxonómica de especies vegetales}

Con base en los usos del suelo del predio y su manejo se realizó el monitoreo del tipo de vegetación, para observar la vegetación predominante o formando congregados alrededor del apiario, y el registro de su hábito de crecimiento. La identificación de las especies melíferas, se realizó en la cuarta y quinta visita al sitio en monitoreo, al distinguir a las especies vegetales que son frecuentadas por las abejas 
en virtud de la oferta de néctar y/o polen, mediante la observación detallada de los especímenes en campo y se verificaron utilizando bibliografía especializada de Lozano y Anhalzer (2013); Aguirre, Yaguana y Merino (2014); Aguirre y Yaguana, (2013), y Valdano (2011).

Se registraron las especies con potencial melífero que se localizaron dentro de un radio de 600-800 m al apiario establecido, cada 30 días durante 4 meses, al observar si se encontraban en floración las especies vegetales, el recurso ofrecido al considerar las pautas propuestas por (Silva y Restrepo, 2012), se observó que las abejas buscan néctar si se encuentran sobre las flores, mientras su abdomen se dilata y se contrae al extraer el néctar de las flores y no se observan bolas de polen en sus corbículas; en cambio en polen si camina sobre toda la flor para desprenderlo de las anteras para que se les pegue en todo el cuerpo, el cual se acumula en las patas traseras, se realiza la verificación de dicha oferta con información de Aguirre y Castañeda (2015); Silva y Restrepo, (2012).

\section{Determinación de la abundancia de la flora melífera}

Se utilizó un procedimiento semi-cuantitativo de acuerdo a la metodología desarrollada por May y Rodríguez, (2012) quienes en su estudio asignaronun valor de 1 a 5, para caracterizar la abundancia, mediante la escala: (1) menos de 1.000 individuos con flores dentro del radio de acción de las abejas, cubriendo menos de $1 \%$ del área, (2) de 1.000 a 10.000 individuos con flores dentro del radio de acción de las abejas, cubriendo menos de 1\% del área, (3) más de 10.000 individuos con flores dentro del radio de acción de las abejas, cubriendo menos de $1 \%$ del área, (4) individuos con flores dentro del radio de acción de las abejas cubren entre 1\% y 5\% del área, y (5) individuos con flores dentro del radio de acción de las abejas cubren más de 5\% del área.

\section{Resultados y Discusión}

\section{A. Plantas melíferas}

En la tabla 2, se indica un resumen general sobre la clasificación taxonómica de las especies vegetales identificadas en el área de estudio, datos sobre hábito de crecimiento, su origen y el recurso ofertado. Los resultados muestran un listado de 32 especies vegetales, pertenecientes a 17 familias botánicas, 
reportadas en la literatura y presentes en la zona de estudio que son las que ofertan recursos florísticos a las abejas. La familia de plantas con mayor cantidad de especies reportadas en la base de datos es la de Fabaceae con 11 especies. Se distingue también por el número de especies las familias: Asteraceae con 3, seguida por las familias; Myrtaceae, Rosaceae y Solanaceae con dos especies. Existen 11 familias botánicas representadas por una especie única (Tabla 2). Resultados a nivel de las familias mejor representadas coincide con estudios similares que reportan a Fabáceas y Asteráceas como las más importantes para otros distritos de la provincia Chaqueña, como se indica en los trabajos de Cabrera y Salgado (2006).

Tabla 2. Listado de plantas nectaríferas y poliníferas presentes en el predio de la Estación Experimental Tunshi

\begin{tabular}{|c|c|c|c|c|c|}
\hline Nombre común & Nombre científico & Familia & Hábito & Origen & R.O \\
\hline Diente de león & Taraxacum officinale & \multirow{3}{*}{ Asteraceae } & Herbácea & Introducido & $\mathrm{N} / \mathrm{P}$ \\
\hline Chilca & Baccharis latifolia & & Arbustivo & Introducido & $\mathrm{N} / \mathrm{P}$ \\
\hline Ñachag & Bidens andicola & & Herbácea & Endémica & $\mathrm{N} / \mathrm{P}$ \\
\hline Falso nabo & Raphanus spp. & Brassicaceae & Herbácea & Introducido & $\mathrm{N} / \mathrm{P}$ \\
\hline Tuna & Opuntia ficus-indica & Cactaceae & Arbustivo & Introducido & $\mathrm{N} / \mathrm{P}$ \\
\hline Sauco & Sambucus nigra & Caprifoliaceae & Arbustivo & Introducido & $\mathrm{P}$ \\
\hline Zambo & Cucurbita fisifolia & Cucurbitaceae & Rastrera & Introducido & $P$ \\
\hline Higuerilla & Ricinus communis & Euphorbiaceae & Arbustivo & Introducido & $\mathrm{N}$ \\
\hline Arveja & Pisum sativum & \multirow{11}{*}{ Fabaceae } & Herbácea & Introducido & $\mathrm{N} / \mathrm{P}$ \\
\hline Trébol blanco & Trifolium repens & & Herbácea & Introducida & $\mathrm{N} / \mathrm{P}$ \\
\hline Lupulina & Medicago lupulina & & Herbácea & Introducido & $\mathrm{N} / \mathrm{P}$ \\
\hline Meliloto & Melilotus indicus & & Herbácea & Introducido & $\mathrm{P}$ \\
\hline Alfalfa & Medicago sativa & & Herbácea & Introducido & $\mathrm{N} / \mathrm{P}$ \\
\hline Falso chocho & Lupinus pubescens & & Herbácea & Introducido & $\mathrm{N} / \mathrm{P}$ \\
\hline Lupina & $\begin{array}{l}\text { Genista } \\
\text { monspessulana }\end{array}$ & & Arbustivo & Introducida & $\mathrm{P}$ \\
\hline Retama & Spartium junceum & & Arbustivo & Introducida & $\mathrm{P}$ \\
\hline Faique & Mimosa quitensis & & Arbustivo & Endémica & $\mathrm{N} / \mathrm{P}$ \\
\hline Retama espinosa & Ulex europaeus & & Arbustivo & Introducida & $\mathrm{N} / \mathrm{P}$ \\
\hline Iso & Dalea coerulea & & Mata & Introducido & $\mathrm{N} / \mathrm{P}$ \\
\hline Romero & Rosmarinus officinalis & Lamiaceae & Arbustivo & Introducido & $\mathrm{N}$ \\
\hline
\end{tabular}

78 Vol. 5, núm. 2, abril 2019, pp.71-93

Miguel Ángel Guallpa Calva, Edmundo Danilo Guilcapi Pacheco, Armando Esteban Espinoza Espinoza 
Dom. Cien., ISSN: 2477-8818

Vol. 5, núm. 2, abril 2019, pp. 71-93

Flora apícola de la zona estepa espinosa Montano Bajo, en la Estación Experimental Tunshi, Riobamba, Ecuador

\begin{tabular}{|l|l|c|c|c|c|}
\hline Eucalipto & Eucalyptus globulus & \multirow{2}{*}{ Myrtaceae } & Arbóreo & Introducido & $\mathrm{N} / \mathrm{P}$ \\
\cline { 5 - 6 } Cepillo blanco & Callistemon viminalis & & Arbustivo & Introducido & $\mathrm{P}$ \\
\hline Cardo santo & Argemone mexicana & Papaveraceae & Mata & Introducido & $\mathrm{P}$ \\
\hline Maíz & Zea mays & Poaceae & Arbustivo & Introducido & $\mathrm{P}$ \\
\hline Lengua de vaca & Rumex obtusifolius & Polygonaceae & Herbácea & Introducido & $\mathrm{N} / \mathrm{P}$ \\
\hline Llánten & Plantago lanceolata & Plantaginaceae & Herbácea & Introducido & $\mathrm{P}$ \\
\hline Capulí & Prunus serotina & \multirow{2}{*}{ Rosaceae } & Arbóreo & Introducido & $\mathrm{N} / \mathrm{P}$ \\
\cline { 5 - 6 } Mora silvestre & Rubus spp & & Arbustivo & Introducido & $\mathrm{N} / \mathrm{P}$ \\
\hline Quishuar & Buddleja incana & \multirow{2}{*}{ Scrophulariaceae } & Arbustivo & Introducido & $\mathrm{N}$ \\
\hline Uvilla & Physalis peruviana & \multirow{2}{*}{ Solanaceae } & Mata & Introducido & $\mathrm{N}$ \\
Hierba mora & Solanum nigrum & & Mata & Introducido & $\mathrm{N} / \mathrm{P}$ \\
\hline Lantana morada & $\begin{array}{l}\text { Lantana } \\
\text { megapotamica }\end{array}$ & \multirow{2}{*}{ Verbenaceae } & Mata & Introducido & $\mathrm{N} / \mathrm{P}$ \\
\hline
\end{tabular}

Fuente: Registros de Trabajo de campo. Elaboración: propia

La familia Asteraceae también fue reportada como una de las más importantes en el estudio de realizado en tres apiarios de la zona rural de Popayán (Vivas, Maca y Pardo, 2008). Resultados que favorecen el manejo del apiario debido a que las abejas prefieren las familias Fabaceae y Asteraceae, porque les proporcionan en la mayoría de los casos, polen y néctar según Pinilla y Nates (2015). La fase inicial de monitoreo, determinó como las principales familias botánicas identificadas con especies nectaríferas y poliníferas en la zona de estudio son; Fabaceae, Asteraceae, Myrtaceae, Rosaceae y Solanaceae.

De acuerdo al origen el 93,75\% de las especies vegetales son introducidas, lo cual tiene sentido dado los siete sistemas de uso del suelo en que basa el manejo del predio, debido a su enfoque investigativoeducativo que se desarrolla en la Estación Experimental Tunshi. Del total de especies identificadas, 4 corresponden a productoras de néctar, mientras que 9 productoras de polen y 19 especies ofertan tanto polen como néctar. En tal sentido, la información generada representa un avance preliminar que requiere ser complementado, a fin de proporcionar alternativas sobre especies vegetales útiles desde el punto de vista apícola. 
Dado que la información de la flora de importancia apícola en la zona es escasa pero muy necesaria, la amplia diversidad vegetal hace que haya múltiples recursos nectaríferos y poliníferos de acuerdo a la zona, que las abejas puedan usar para obtener una gama de tipos y calidades de miel de abeja (Zavala-Olalde et al., 2013). Por ello, al disponer de información acerca de las plantas que más tributan a la producción de miel permitirá planear mejor el manejo de las colmenas, favorecerá la valoración, y con ello la conservación y/o restauración de la cobertura vegetal. Con el propósito de contribuir al mejoramiento de las condiciones técnicas del apiario, la producción, y la calidad de la miel (Hoyos, 2007).

\section{B. Descripción de especies vegetales del estudio}

Se caracterizó a todas las especies melíferas identificadas, mediante una descripción rápida, considerando su taxonomía, uso y morfología como se presenta a continuación:

Nombre científico: Taraxacum officinale Weber

Familia: Asteraceae

Usos: Forrajera, medicinal

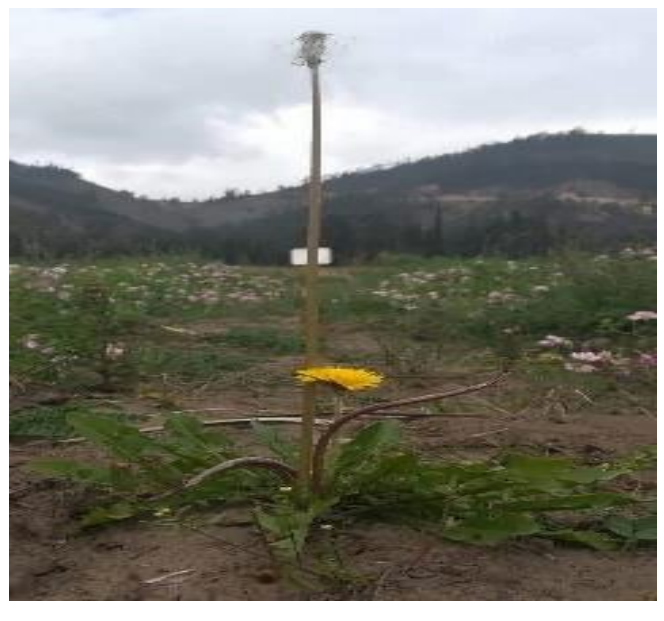

Morfología: Hierba siempre verde. Raíz subterránea vertical, cónica, larga y muy ramificada, de sabor agridulce. Hojas de sabor amargo, que salen de la raíz en roseta, de márgenes dentados, de tamaño muy variable, de unos 5 - 30 $\mathrm{cm}$ de largo por1 - $5 \mathrm{~cm}$ de ancho. Flores en capítulos solitarias con tallo igual o algo más largos que las hojas, amarillas, hermafroditas. Fruto es un aquenio cilíndrico, seco de color pardo o rojizo (Medicamentos herbarios tradicionales, 2009).

Imagen 1. Diente de león 
Nombre científico: Baccharis latifolia (Ruiz \& Pav.) Pers.

Familia: Asteraceae

Usos: Medicinal, recuperación de suelos, protección de taludes.

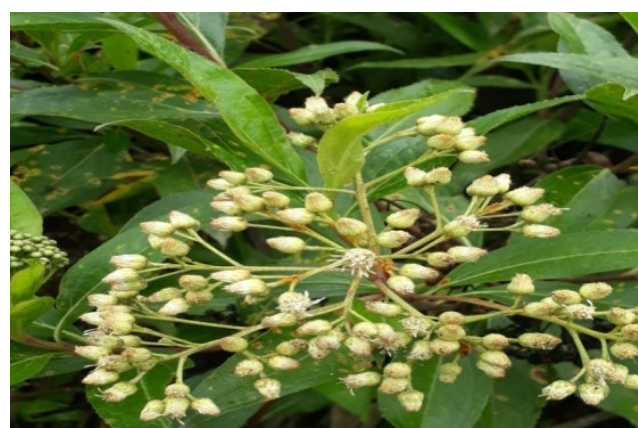

Morfología: Arbusto muy ramificado. Sus hojas simples, alternas, de borde dentado, de forma lanceolada, su color verde brillante. Inflorescencias en panículas terminales ramificada. Frutos en aquenios (Aguirre y Yaguana, 2013).

Imagen 2. Chilca

Nombre científico: Bidens andicol aKunth

Familia: Asteraceae

Usos: Medicinal

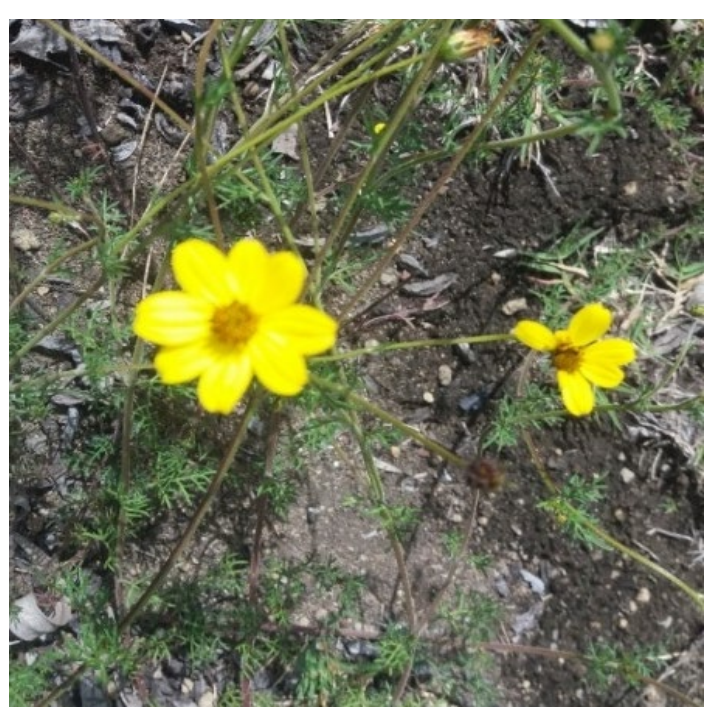

\section{Morfología:}

Hierba perenne. Los tallos de hasta $40 \mathrm{~cm}$ de largo, numerosos y ramificados, estriados, a veces con pubescencias. Hojas simples, opuestas (las superiores a veces alternas). Inflorescencia en numerosas cabezuelas solitarias, ubicadas en el ápice de los tallos. Flores pequeñas sésiles dispuestas sobre un receptáculo convexo. Fruto un aquenio seco indehiscente, negruzcos, contiene una sola semilla.

(Aguirre, Yaguana y Merino, 2014).

Imagen 3. Ñachag 
Nombre científico: Rhapannus spp.

Familia: Brassicaceae

Usos: Alimenticio, forrajera

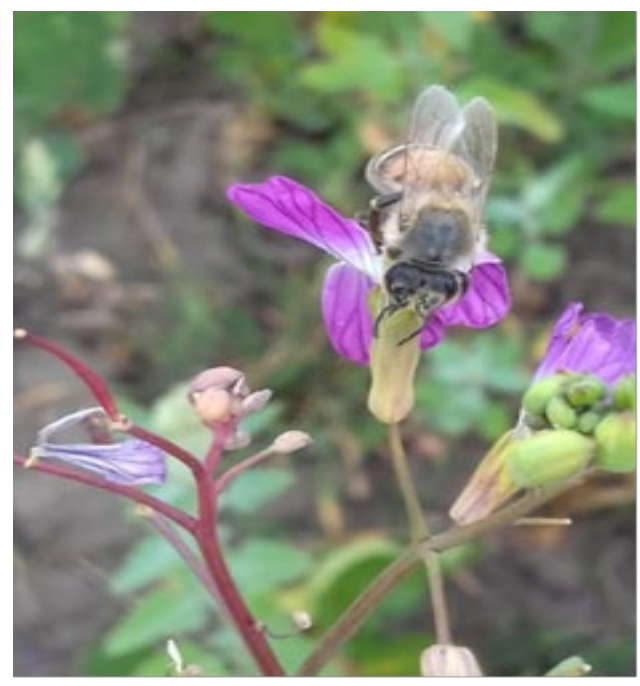

Morfología: Planta anual de 50 a $80 \mathrm{~cm}$ de altura, con raíz napiforme, de tallo herbáceo, con escapo reducido durante el desarrollo vegetativo y alargado durante el periodo reproductivo. En el primer ciclo: hojas basales, lobuladas irregularmente, alternas y hojas superiores oval-lanceoladas, alternas, sésiles más pequeñas que las basales para el segundo ciclo. Flores en racimos terminales, con cuatro pétalos morados en cruz. Fruto una silicua cilíndrica alargada (Mazorca, 1979).

\section{Imagen 4. Falso nabo}

Nombre científico: Opuntia ficus-indica (L.) Mill.

Familia: Cactaceae

Usos: Alimenticio, medicinal

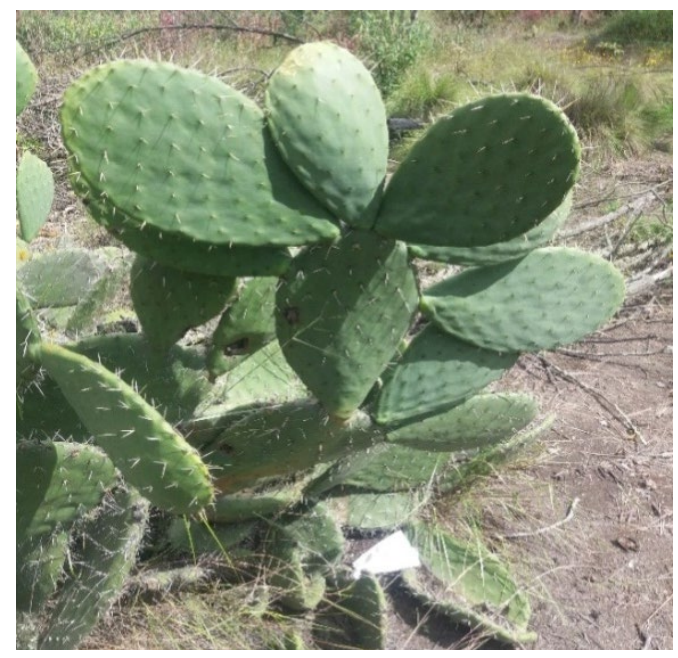

Morfología: Planta arbustiva de 3 a $5 \mathrm{~m}$ de alto, los segmentos o cladodiosenque se divide, son tallos capaces de ramificarse, emitiendo flores o frutos. Sus hojas son hojuelas formadas por espinas que salen desde una areola, las flores son solitarias de color amarillo dependiendo de la especie, $\mathrm{Su}$ fruto es una baya polisperma de forma ovoide esférica de color verde y toma diferentes colores cuando maduran, son comestibles, agradables y dulces (Castro, 2009).

Imagen 5. Tuna 


\section{Nombre científico: Sambucus nigra L.}

Familia: Caprifoliaceae

Usos: Agroforestal, ornamental, medicinal.

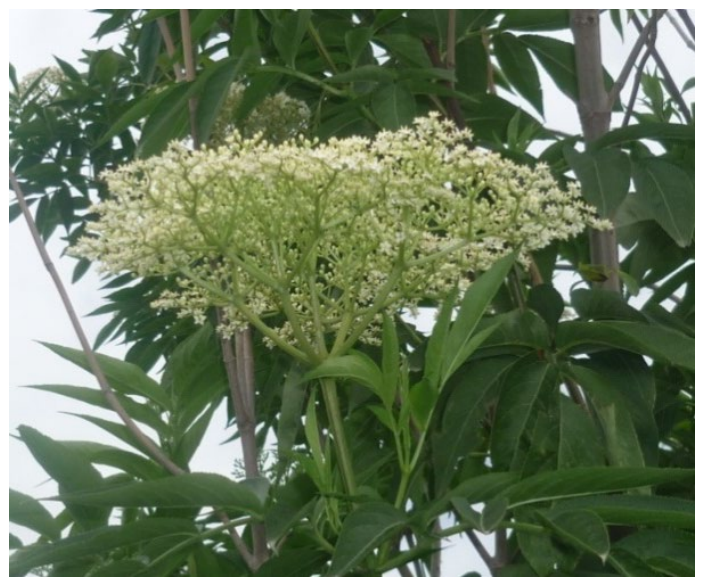

Morfología: Árbol de follaje anual, de hasta 5 - $6 \mathrm{~m}$ de altura. Corteza pardo-grisácea. Ramas cenicientas con médula blanquecina bien desarrollada. Hojas opuestas, compuestas. Flores pequeñas, blancas, muy aromáticas, dispuestas en un conjunto que asemeja un paraguas. Fruto redondeado de $3-5 \mathrm{~mm}$ de diámetro, de color negro violáceo y sabor agradable, con tres semillas en su interior (Ruiz, 2007).

Imagen 6. Tilo

\section{Nombre científico: Cucurbita fisifolia}

Familia: cucurbitaceae

Usos: Forraje

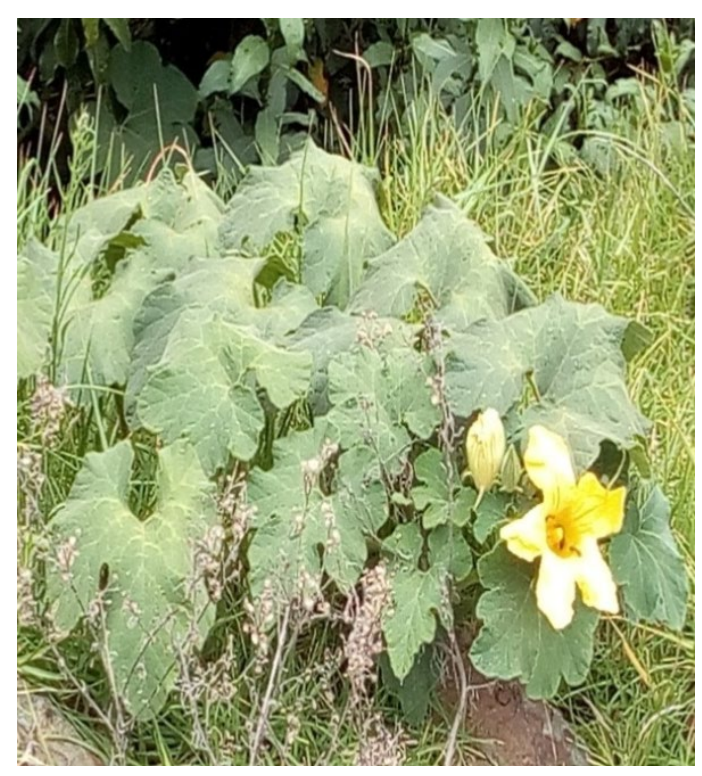

Morfología: Especie rastrera o trepadora, de tallo cubierto de vellosidades. Hojas alternas, ampliamente ovadas a casi circulares, de hasta $25 \mathrm{~cm}$ de largo y de ancho, de base acorazonada, el margen ondulado-dentado, cubiertas de tricomas, los pecíolos de hasta $26 \mathrm{~cm}$ de largo. Las flores son solitarias en las axilas de las hojas. Las masculinas sobre largos pedúnculos y las femeninas con pedúnculos cortos de hasta $5 \mathrm{~cm}$ de largo. Los frutos globosos a oblongos, de hasta $35 \mathrm{~cm}$ de largo y $20 \mathrm{~cm}$ de ancho de color verde con rayas longitudinales de color crema (Stevens et al., 2001).

Imagen 7. Zambo 


\section{Nombre científico: Ricinus communis}

Familia: Euphorbiaceae

Usos: ornamental, medicinal

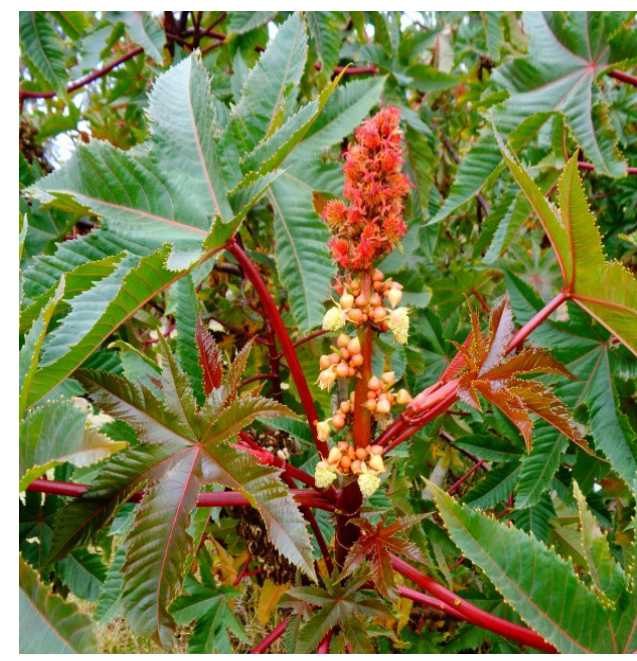

Es una planta perenne, presenta un tallo principal recto, seccionado por entrenudos. Hojas alternas, pecioladas, palmeadas con 5 a 11 lóbulos, dentadas, con nerviación palmatinervia, con peciolos redondos de 18 a $60 \mathrm{~cm}$ de longitud. Inflorescencia en racimo, es una planta monoica y generalmente unisexual. Su fruto es una cápsula globosa (Robles, 1980).

Imagen 8. Higuerilla

Nombre científico: Pisum sativumL.

Familia: Fabaceae

Usos: Alimenticio

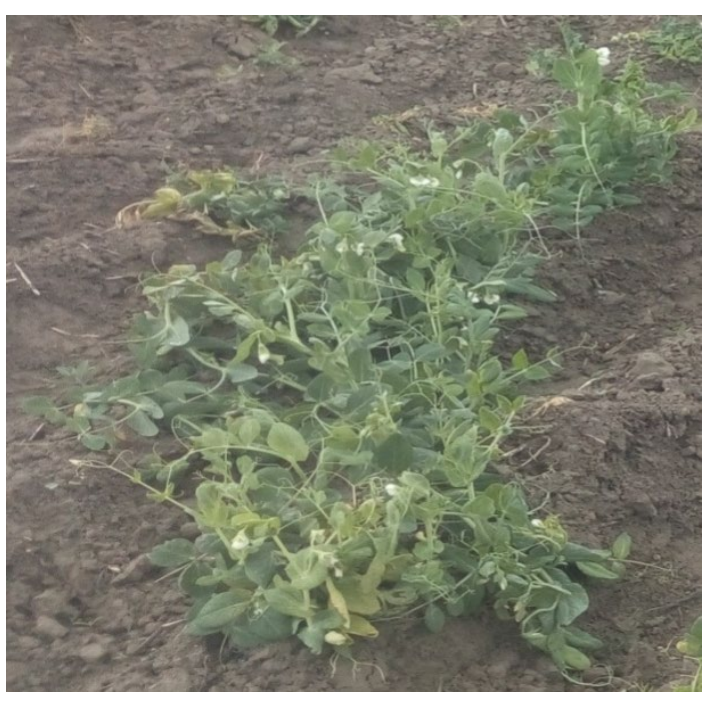

Es planta anual herbácea, los tallos son trepadores y angulosos. Las hojas tienen pares de foliolos y terminan en zarcillos, que tienen la propiedad de asirse a los tutores que encuentran en su crecimiento. Las vainas tienen de 5 a $10 \mathrm{~cm}$ de largo y suelen tener de 4 a 10 semillas. Las semillas de arveja tienen una ligera latencia. Desde que nacen las plantas hasta que se inicia la floración, cuando las temperaturas son óptimas, suelen transcurrir entre $90 \mathrm{y}$ 140 días, según variedades (AGRO, 2012).

Figura 9. Arveja 


\section{Nombre científico: Trifolium repens L.}

Familia: Fabaceae

Usos: Forraje

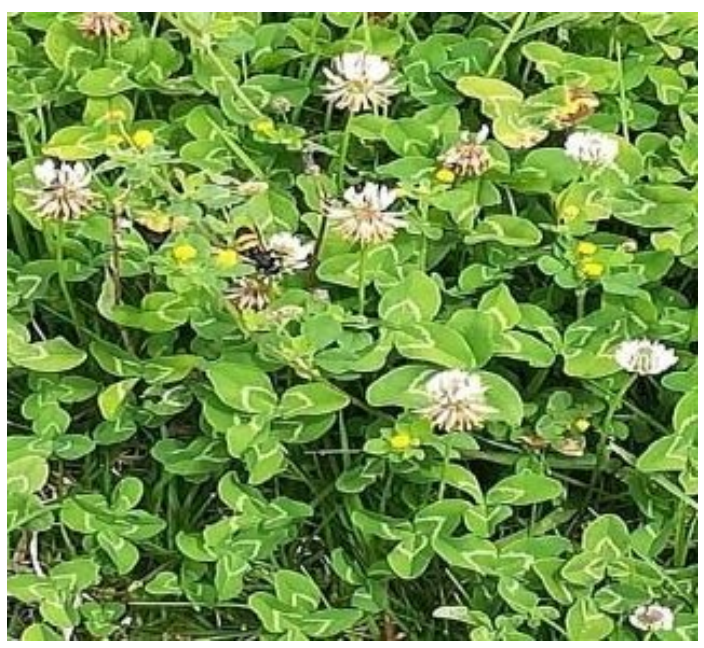

Morfología: Planta perenne de 10-50 cm. Tallos rastreros y enraizantes. Hojas trifoliadas, foliolos obovados, denticulados, a menudo con una mancha blanca en el haz. Estípulas bruscamente estrechadas en el ápice. Flores con corola blanca o rosada, membranosa en la fructificación; presentan una pequeña bráctea en su base. Cáliz con 10 nervios. Flores agrupadas en cabezuelas globosas pedunculadas (Ratera, 1991).

Imagen 10.Trébol blanco

\section{Nombre científico: Medicago lupulina}

\section{Familia: Fabaceae}

Usos: Como alimento para cuyes, conejos y vacas.

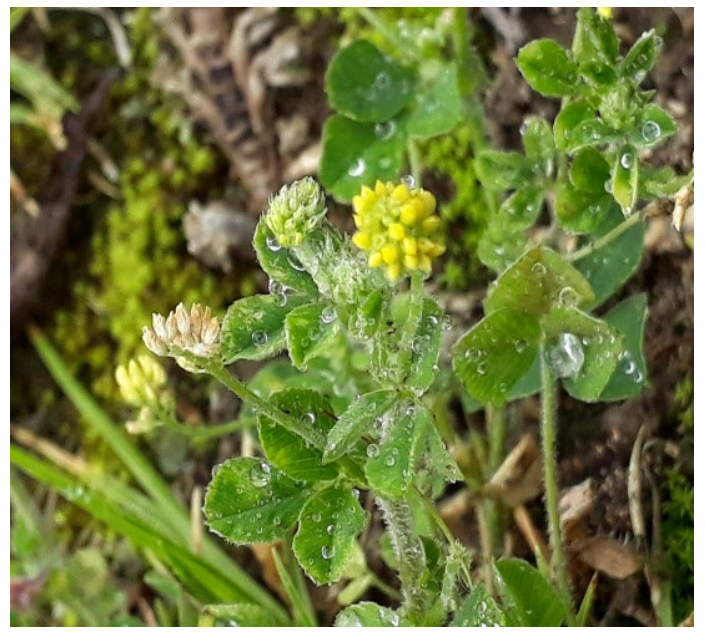

Morfología: Hierba perenne de raíz axonomorfa, corta, con presencia de nódulos bacterianos. Tallo rastrero de ramificación basítona. Hojas alternas, pecioladas, compuestas, trifoliadas, con los foliolos ovales, aserrados, mucronados y pubescentes. Sus flores en racimos cortos pequeños, de color amarillo. Fruto es una vaina pequeña de una sola semilla, de color negro cuando madura (Valdano, 2011).

Imagen 11. Lupulina

\section{Nombre científico: Medicago sativa}

Familia: Fabaceae

Usos: Forrajera, alimenticia, medicinal

85 Vol. 5, núm. 2, abril 2019, pp.71-93

Miguel Ángel Guallpa Calva, Edmundo Danilo Guilcapi Pacheco, Armando Esteban Espinoza Espinoza 


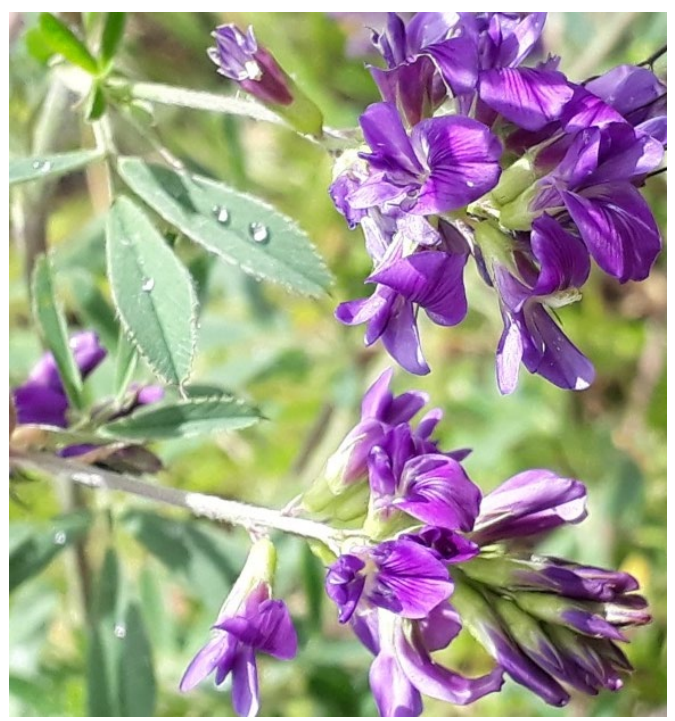

Morfología: Es una planta plurianual, herbácea, con hojas trifoliadas y con el margen aserrado. Sus flores son de color violeta a purpura las inflorescencias numerosas se agrupan en racimos densos terminales. Estas flores dan lugar a semillas en vainas con forma de espiral (Rosario, 2015).

Imagen 12. Alfalfa

Nombre científico: Lupinus pubescens Benth

Familia: Fabaceae

Usos: Forrajera, alimenticio.

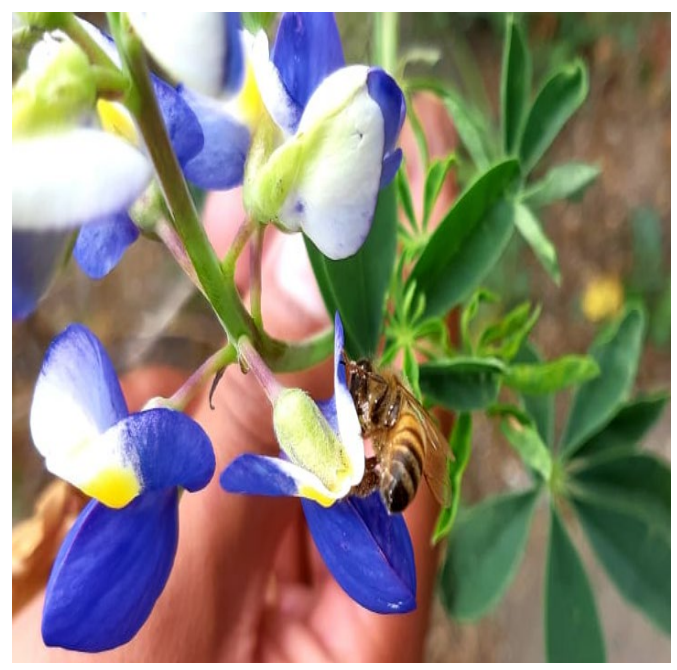

Planta perenne, de hasta $80 \mathrm{~cm}$ de alto, pubescente, con hojas compuestas en grupos de hasta 9 foliolos, oblongoslanceolados. Inflorescencia racimosa, axilar. Flores de color violeta intenso. El fruto es una legumbre. Semillas usualmente aplanadas (Fundación Bótanica de los Andes , 2017 ).

\section{Abundancia de las especies vegetales del estudio.}

En la tabla 3, se distingue la cantidad de individuos por especie vegetal que estuvieron en floración durante el periodo de evaluación. Siendo el área de cultivos, donde se estimó más de 10.000 individuos 
Dom. Cien., ISSN: 2477-8818

Vol. 5, núm. 2, abril 2019, pp. 71-93

con flores de Raphanus spp. Y de 1.000 a 10.000 individuos se registró a Lupinus pubescens y Zea mays, con similar valor se determinó aBidens andicolay Eucalyptus globulus presentes en los sistemas de uso; rastrojo y bosque plantado respectivamente. El resto de especies vegetales presentaron una ocupación con menos de 1.000 individuos con flores presentes (1). Desde dos especímenes en el bosque plantado hasta siete en los sistemas agroforestales.

Tabla 3. Abundancia de flora melífera

\begin{tabular}{|c|c|c|c|c|c|c|c|c|}
\hline Nombre científico & Nombre local & 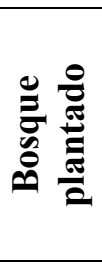 & $\frac{\mathscr{0}}{\mathscr{0}}$ & 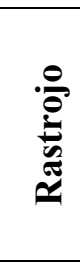 & 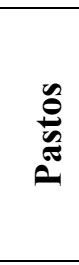 & 苞 & 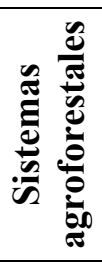 & 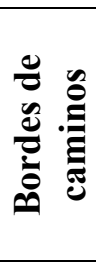 \\
\hline Taraxacum officinale & Diente de león & & & & 1 & & & \\
\hline Baccharis latifolia & Chilca & & 1 & & & & & \\
\hline Bidens andicola & Nachag & & & 2 & & & & 1 \\
\hline Raphanus spp. & Falso nabo & & & & & 3 & & \\
\hline Opuntia ficus-indica & Tuna & & & & & 1 & & \\
\hline Sambucus nigra & Tilo & & & & & & & 1 \\
\hline Cucurbita fisifolia & Zambo & & 1 & & & & & \\
\hline Ricinus communis & Higuerilla & & & 1 & & & & 1 \\
\hline Pisum sativum & Arveja & & & & & 1 & & \\
\hline Trifolium repens & Trébol blanco & & & & 2 & & & \\
\hline Medicago lupulina & Lupulina & & & & 1 & & & \\
\hline Melilotus indicus & Meliloto & & & & 1 & & & \\
\hline Medicago sativa & Alfalfa & & & & 1 & & & \\
\hline Lupinus pubescens & Falso chocho & & & & & 2 & & \\
\hline
\end{tabular}

Abundancia: (1) menos de 1.000 individuos con flores dentro del radio de acción de las abejas, (2) de 1.000 a 10.000 individuos con flores dentro del radio de acción de las abejas, cubriendo menos de 1\% del área, (3) más de 10.000 individuos con flores dentro del radio de acción de las abejas, cubriendo menos de $1 \%$ del área.

\section{Conclusiones}

En la Estación Experimental Tunshi, las familias de mayor importancia melífera son Asteraceae, seguida por las familias; Myrtaceae, Rosaceae y Solanaceae.Apis mellifera tiene la oferta de recursos apícolas, de alrededor de 32 especies vegetales identificadas en el predio. Para el fortalecimiento de 
la apicultura en la zona en estudio, es imperante el manejo del bosque plantado de Eucalipto y de las restantes unidades de manejo que conforman el predio, más el aumento de las poblaciones de especies de interés apícola de procedencia nativa que contribuyan a la producción, mediante su plantación en diversas formas de sistemas agroforestales.

Los resultados del listado de especies melíferas del presente estudio son preliminares, aún se requiere complementar con información de su abundancia y su época e intensidad de floración para un periodo de al menos un año de monitoreo de tal forma que se pueda establecer interacciones entre las variables de manejo de las unidades de uso del predio y las del tipo climático predominantes del sitio evaluado.

\section{Referencias Bibliográficas}

AGRO, E., 2012. La arveja y clima en el Ecuador. El Agro.

Aguirre, Z., Yaguana C. Y Merino B., 2014. Plantas medicinales de la zona andina de la provincia de Loja. Primera Edición. Loja, Ecuador. 193 p.

Aguirre S., M., Castañeda S., T., 2015. Identificación palinológica en mieles de la costa chica de Guerrero y Oaxaca. Tesis licenciatura. Unidad Académica de Medicina Veterinaria y Zootecnia, Universidad Autónoma de Guerrero, 84p.

Aguirre, Z., y Yaguana, C., 2013. Árboles y arbustos de parques y avenidas de Loja.

Bischeimer, M. 2012. Flores de la Patagonia Argentina. Flores nativas y exóticas presentes en los ambientes cordilleranos y precordilleranos de la Patagonia argentina.Argentina - Patagonia: Serie Patagonia.

Añazco, M., 2008. “Usos medioambientales de las plantas”. In: de la Torre, L., H. Navarrete, P. Muriel M., M. J. Macía \& H. Balslev (eds.). Enciclopedia de las Plantas Útiles del Ecuador. Herbario QCA de la Escuela de Ciencias Biológicas de la Pontificia Universidad Católica del Ecuador \& Herbario AAU del Departamento de Ciencias Biológicas de la Universidad de Aarhus. Quito, EC \& Aarhus. Consultado el 20 de noviembre de 2018. Disponible en http://www.biologia.puce.edu.ec/imagesFTP/10457.Medioambiental.pdf 
Brophy, J. C., 2013. Melaluecas, their botany, essential oils and uses.

Cabrera, M. M. y Salgado, C. R., 2006. “Contribución al estudio de la flora melífera de la provincia de Formosa, Argentina”. UNNE. Comunicaciones Científicas y Tecnológicas 2006. Resumen: B-001

Calvache, J., 2016. La investigación científica como alternativa en la formación profesional. Colombia: CEPUN.

Castro, J., 2009. Cultivo de la tuna (Opuntia ficus indica). Trujillo - Peru.

Castro, J; Cerdas, M., 2005. Mora (Rubus spp) Cultivo y Manejo Poscosecha. San José Costa Rica. MAG.

Decourtye, A., Mader, E. y Desneux, N., 2010. Landscape enhancement of floral resources for honey bees in agro-ecosystems. Apidologie, 41: 264-277.

Di Marco, E., 2015. Eucalyptus globulus sp. globulus Labill. Direccion de produccion forestal.

Estación Meteorológica Guaslán 2016. Datos climáticos de la serie 2013-2016.Riobamba.

Fischer, G., Flores, V., \& Sora, A., 2000. Producción, poscosecha y exportación de lauchuva (Physalis peruviana L.). .Bogotá - Colombia.

Fischer, G., \& Miranda, D., 2011. Uchuva (Physalis peruviana L.). En: Manual defrutales. Bogotá: Produmedios.

Heywood, V., y R, W., 1995. Global Biodiversity assessment. UNEP, Cambridge Univ. Press.

INIAP. (2011). Boletín divulgativo N406 “Guía para la producción de maíz en la sierra sur del Ecuador”. Programa de Maíz. EESC. Quito-Ecuador. 1-3pp.

Fundación Bótanica de los Andes . 2017. Plantas Nativas de la Hoya de Quito . Obtenido de Lupinus pubescens: http://plantasnativas.visitavirtualjbq.com/index.php/emblematicas/4-lupinuspubescens

89 Vol. 5, núm. 2, abril 2019, pp.71-93

Miguel Ángel Guallpa Calva, Edmundo Danilo Guilcapi Pacheco, Armando Esteban Espinoza Espinoza 
Hoyos, D., 2007. Manejo sostenible de la producción de miel de abejas para el pequeño productor. Tesis de pregrado, Universidad de la Salle Gerencia de Empresas Agropecuarias, Bogotá, Colombia.

Instituto Interamericano de Cooperación para la Agricultura. IICA., Ministerio de Agricultura. MG., 2009. Manual de Apicultura Básica para Honduras. Tegucigalpa

Instituto Nacional de Tecnología Agropecuaria. INTA. 2018. Manual de Apicultura. Buenos Aires, Argentina.

Jarmillo, H., 2008. Inventario Florístico del sector de Buga Bajo del bosque del Paquiestancia Cayambe. Cayambe.

López, C., Odorizzi, A., Basigalup, D., Arolfo, V., \& Martínez, M., 2016. El trébol de olor blanco y su uso en la provincia de Córdoba. Buenos Aires: INTA.

Lozano, P., \& Anhalzer, J., 2013. Flores silvestres del Ecuador. (2 $2^{\text {da }}$ ed.). Ecuador: Mariscal

May, T., \& Rodríguez, S., 2012. Plantas de interés apícola en el paisaje: Observaciones de campo y la percepción de apicultores en República Dominicana. Revista Geográfica de América Central, 48 (1), 133-162.

Mazorca, A., 1979. Manual de malezas. Buenos Aires: Hemisferio Sur. Medicamentos herbarios tradicionales, MHT., 2009. 103 Especies vegetales. Santiago.

Muñoz, C., 2002. Plantas Medicinales Españolas Rosmarinus officinalis. Biblid.

Naturalista. 2016. Argemone mexicana. Consultado en julio de 2016 en: http://naturalista.conabio.gob.mx/taxa/128533-Argemonemexicana\#Taxonom.C3.ADa

Ocaña, R y Ocaña, P., 2008. Prácticas de Apicultura.

Padilla, S., 1995. Manejo agroforestal andino. Quito - Ecuador. 
Pérez, J., 2004. Extraccion de Proteasas de Ulex europaeus L. y su potencial urtilizacion como sustituto de cuajo. Universidad Austral de Chile.

Pinilla-Gallego, M. y Nates-Parra, G., 2015. Diversidad de visitantes y aproximación al uso de nidos trampa para Xylacopa (Hymenoptera: Apidae) en una zona productora de pasiflora en Colombia. Actualidades Biológicas, 37(103), 143-153.

Ratera, M., 1991. Praderas y forrajes: Producción y aprovechamiento. Madrid: Mundi-Prensa.

Robles, S., 1980. Producción de oleaginosas y textiles. Mexico - Mexico: Limusa.

Rosario, M., 2015. Medicago sativa: mejora de la productividad y nuevos aspectos de su valor nutritivo y funcional. Universidad de Granada.

Romoleroux, K., 1996. Flora of Ecuador, Rosaceae No 56. Edited by Harling and Andersson. Berlings, Arlöv, Sweden.

Ruiz, T., 2007. Flora Ibérica. Plantas vasculares de la Península Ibérica e Islas Baleare. Madrid España.

Sánchez, S., 1995. Calendarios Apícolas para el Suroeste Antioqueño. Miscelánea Sociedad Colombiana de Entomología. Comité Seccional de Antioquia. Trabajos de investigación sobre abejas africanizadas. Fase II No. 32 Mayo.

Sánchez, C., 2013. Crianza y producción de abejas-apicultura. Perú: Ripalme.

Sierra, R., 2012. Propuesta preliminar de un sistema de clasificación para el Ecuador Continental. Quito, Ecuador: Proyecto INEFAN/GEF-BIRF y EcoCiencia.

Silva, L; y Restrepo, S., 2012. Flora Apícola. Determinación de la oferta floral apícola como mecanismo para optimizar la producción, diferenciar producto de la colmena y mejorar la competitividad. Instituto de investigación de recursos biológicos Alexander von Humboldt. 
Stevens, W. D., C. Ulloa U., A. Pool y Montiel, O.M., (eds.), 2001. Flora de Nicaragua. Vol. 85, tomos I, II y III. Missouri Botanical Garden Press. St. Louis, Missouri.

Tolosa, H., 2014. Flora Bonaerense. Plantas y Hongos de la provincia de Buenos Aires, Argentina. Obtenido de: https://florabonaerense.blogspot.com/2014/12/camara-morado-lantanamegapotamica.html

Valdano, T., 2011. Plantas alto andinas del Ecuador. Quito-Ecuador: Editorial Universitaria AbyaYala.

Vibrans, H., 2009. Malezas de Mexico. Mexico.

Vivas, N., Maca, J., \& Pardo, M., 2008. Caracterización cualitativa del polen recolectado por Apis mellifera L en tres apiarios del municipio de Popayán. Facultad de ciencias Agropecuarias, 6 (2), 94-98.

Vivas , J., 2015. Prevalencia de Nosema (Nosema spp.) en Colmenares de la Región Norte y Centro Norte del Ecuador. Recuperado el 12 de Enero de 2019, de http://www.dspace.uce.edu.ec/bitstream/25000/7811/1/T-UCE-0004-62.pdf

Zavala-Olalde, A., Colmo-González, I, Matalí-Pérez, N., Piana, L., Olivier, B., Méndez Villarreal, A., yVandame, R., 2013. Characterization of four typical honeys from highly diverse tropical ecosystems.Journal of Apicultural Research, 52(2):24-34. 
Dom. Cien., ISSN: 2477-8818

Vol. 5, núm. 2, abril 2019, pp. 71-93

REVISTA

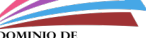

LAMINIO DE CIENCIAS

Flora apícola de la zona estepa espinosa Montano Bajo, en la Estación Experimental Tunshi, Riobamba, Ecuador

93 Vol. 5, núm. 2, abril 2019, pp.71-93

Miguel Ángel Guallpa Calva, Edmundo Danilo Guilcapi Pacheco, Armando Esteban Espinoza Espinoza 